\title{
Neutrino phenomenology and unparticle physics
}

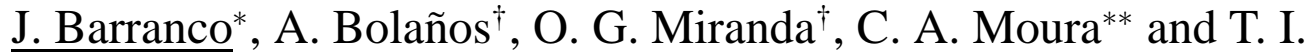 \\ Rashba \\ *Max-Planck-Institut für Gravitationsphysik (Albert-Einstein-Institut), Am Mühlenberg 1, D-14476 \\ Golm, Germany \\ ${ }^{\dagger}$ Departamento de Física, Centro de Investigación y de Estudios Avanzados del IPN, Apartado \\ Postal 14-740 07000 México, D F, México \\ ** INFN Sezione di Napoli, Complesso Universitario Monte S. Angelo, Via Cintia, I-80126 Napoli, \\ Italy \\ ${ }^{\ddagger}$ Max-Planck-Institute for Solar System Research, Katlenburg-Lindau, 37191, Germany and \\ IZMIRAN, Troitsk, Moscow region, 142190, Russia
}

\begin{abstract}
We show how neutrino data can be used in order to constrain the free parameters of possible extensions to the standard model of elementary particles (SM). For definiteness, we focus in the recently proposed unparticle scenario. We show that neutrino data, in particular the MUNU experiment, can set stronger bounds than previous reported limits in the scale dimension parameter for certain region $(d>1.5)$. we compute the sensitivity of future neutrino experiments to unparticle physics such as future neutrino-electron scattering detectors, coherent neutrino-nuclei scattering as well as the ILC . In particular, we show that the measurement of coherent reactor neutrino scattering off nuclei provide a good sensitivity to the couplings of unparticle interaction with neutrinos and quarks. Finally our results are compared with the current astrophysical limits.
\end{abstract}

PACS: $13.15 .+\mathrm{g}, 12.90 .+\mathrm{b}, 23.40 . \mathrm{Bw}$

\section{THE $v-$ SM}

The standard model of elementary particles (SM) is perhaps the most successful model that has been experimentally tested. Nevertheless, the so called solar and atmospheric neutrino problems jeopardized the effectiveness of the SM. It took around 30 years of intense debate and confrontation of theory and experiments to realize that the correct mechanism needed to understand the deficit between expected neutrinos and the measured events at terrestrial detectors is the neutrino oscillation mechanism [1]: neutrinos change their flavor due to the fact that they are massive and their flavor states are a mixture of their mass states. This is the first evidence of physics beyond the SM and consequently it requests to extend the SM in the leptonic sector. In summary, SM needs to incorporate:

- Neutrinos masses $m_{v 1}, m_{v 2}, m_{v 3}$ and,

- mixing in leptonic sector, that is, an equivalent to the CKM matrix called $U_{P M N S}\left(\theta_{i j}\right)$, where the matrix $U_{P M S}$ is the mixing matrix between flavor states and mass states, i.e. $\left|v_{\alpha}\right\rangle=\sum_{a} U_{\alpha a}^{*}\left|v_{a}\right\rangle, a=1 . .3, \alpha=e, u, \tau$.

This will be refereed as the $v$-SM. The current values for the masses and mixing angles can be found elsewhere [2]. The objective of the present note, as part of the Proceeding of the CINVESTAV's Advanced Summer School, is to illustrate how neutrino phe- 
nomenology, within the $v-\mathrm{SM}$, can constrain others possible extensions to the SM. As an example, we will focus on the recently proposed unparticle scenario [3, 4], where a scale invariant sector could exist above TeV energies that can couple with the SM sector in the low-energy limit. For the student's benefit, in this note we present a general overview of how constrain nonstandard properties with neutrino. For a more detailed discussion on the unparticle's parameter limits by using the latest reactor neutrino data, we suggest to read [5].

\section{CONSTRAINING NEW PHYSICS WITH NEUTRINO DATA}

It turns out that the biggest difficulty for detecting neutrino is at the same time the biggest advantage: the neutrino interacts only through weak interactions. Within the $v-\mathrm{SM}$, the neutrino doesn't have electric charge, or magnetic moment and any other interaction will be automatically considered as nonstandard interaction. That makes them the perfect candidate for detecting physics that is not considered within the SM because most of the times, in the low energy limit, those extension will produce new charged or neutral currents, flavor neutral changing currents (FCNC), or new neutrino properties. This situation can be easily translated into an algorithm for constraining the parameters of a possible SM's extension:

1. Star with $v$-SM: SM+neutrino masses and mixing. Compute standard processes.

2. Propose a new theory: derive a new effective Lagrangian at low energies, $\mathscr{L}^{N E W}(\varepsilon, \eta, \ldots)$, that introduces new parameters besides those considered in the SM.

3. Compute physical processes: either scattering or decay processes $\left(\frac{d \sigma^{N e w}}{d T}, \Gamma^{N e w}\right)$.

4. Impose limits on the free parameters by comparing with experimental data through an statistical analysis (usually using a $\chi^{2}$ analysis).

For instance, the step number 3 can be directly be used to compute the expected number of neutrinos. Neglecting for a moment the detector efficiency and resolution, it can be computed as

$$
N_{\text {events }}^{\text {Theo }}(\eta, \varepsilon \ldots)=t \phi_{0} \frac{M_{\text {detector }}}{M} \int_{E_{\min }}^{E_{\max }} d E_{v} \int_{T_{t h}}^{T_{\max }\left(E_{v}\right)} d T \lambda\left(E_{v}\right) \frac{d \sigma}{d T}\left(E_{v}, T, \eta, \varepsilon \ldots\right),
$$

with $t$ the data taking time period, $\phi_{0}$ the total neutrino flux, $M_{\text {detector }}$ the total mass of the detector, $\lambda\left(E_{V}\right)$ the normalized neutrino spectrum, $E_{\max }$ the maximum neutrino energy and $T_{t h}$ the detector energy threshold. The contribution due to new physics is encapsulated in the total cross section

$$
\frac{d \sigma\left(E_{v}, T, \eta, \varepsilon \ldots\right)}{d T}=\frac{d \sigma^{S M}\left(E_{v}, T\right.}{d T}+\frac{d \sigma^{N e w}\left(E_{v}, T, \eta, \varepsilon\right)}{d T}
$$

which contains both, the SM and the new physics contribution obtained from $\mathscr{L}^{N E W}(\eta, \varepsilon \ldots)$ including also the interference terms, if they are not absent. For the step 
TABLE 1. Cross section for the relevant SM processes. Here $G_{F}$ is the Fermi constant, $g_{V}, g_{A}, g_{v}^{n, p}, g_{A}^{n, p}$ are the SM coupling constants to electron, protons and neutrons respectively, $M_{e}$ the electron mass, $M$ the nucleon mass, $G_{V}=\left[g_{V}^{p} Z+g_{V}^{n} N\right] F_{n u c l}^{V}\left(Q^{2}\right), G_{A}=$ $\left[g_{A}^{p}\left(Z_{+}-Z_{-}\right)+g_{A}^{n}\left(N_{+}-N_{-}\right)\right] F_{n u c l}^{A}\left(Q^{2}\right) . F_{n u c l}^{V}\left(Q^{2}\right)$ is the nuclear form factor, $Z$ the atomic number, and $N$ the number of neutron in the nuclei. \pm in $N, Z$ refers to the polarization.

\begin{tabular}{cc}
\hline$v-\mathrm{SM}$ process & Cross section \\
\hline$v_{e}+e \rightarrow v_{e}+e$ & $\frac{d \sigma\left(E_{v}, T\right)}{d T}=\frac{G_{F}^{2} M_{e}}{2 \pi}\left(\left(g_{V}+g_{A}\right)^{2}+\left(g_{v}-g_{A}\right)^{2}\left(1-\frac{T}{E_{v}}\right)^{2}-\left(g_{v}^{2}-g_{A}^{2}\right) m_{e} \frac{T}{E_{v}}\right)$ \\
$e^{+}+e^{-} \rightarrow v \bar{v}+\gamma$ & $\sigma_{\mathrm{LEP}}^{\text {theo }}(s)=\int \mathrm{d} x \int \mathrm{d} c_{\gamma} \frac{2 \alpha}{\pi x s_{\gamma}}\left[\left(1-\frac{x}{2}\right)^{2}+\frac{x^{2} c_{\gamma}^{2}}{4}\right] \sigma_{0}^{\text {theo }}(\hat{s})$ \\
Coherent $v+N \rightarrow v+N$ & $\frac{d \sigma}{d T}=\frac{G_{F}^{2} M}{2 \pi}\left\{\left(G_{V}+G_{A}\right)^{2}+\left(G_{V}-G_{A}\right)^{2}\left(1-\frac{T}{E_{v}}\right)^{2}-\left(G_{V}^{2}-G_{A}^{2}\right) \frac{M T}{E_{v}^{2}}\right\}$ \\
\hline
\end{tabular}

number 4 , the comparison of the theoretical expected events and the experimentally observed can be done by computing and minimizing the $\chi^{2}$ function

$$
\chi^{2}(\eta, \varepsilon \ldots)=\frac{\left(N_{\text {events }}^{\text {Exp }}-N_{\text {events }}^{\text {Theo }}(\eta, \varepsilon \ldots)\right)^{2}}{\delta N_{\text {events }}^{2}},
$$

which automatically give us the allowed region for the free parameters $\{\eta, \varepsilon \ldots\}$ at some confidence level.

\section{THE CASE OF UNPARTICLE PHYSICS}

Neutrino data can offer the possibility of studying unparticle phenomenology in two ways: first one by studying the effects of virtual unparticle exchanged between fermionic currents, second one by studying the direct production of unparticles. The neutrinoelectron and neutrino-nuclei scattering are examples where unparticle effects of the first type are measurable, while single-photon production $\left(e^{-} e^{+} \rightarrow \gamma X\right)$ at LEP is an example of direct production of unparticles. Notice that, beside neutrinos $(v \bar{v}), X$ can be any new hypothetical particle, in particular, unparticle stuff. In this case, neutrino production is the background reaction, because the signatures for detection of unparticles are also the missing energy and momentum.

Now let us perform our algorithm for the particular case of the unparticle scenario.

- Step 1: Starting with the $v-\mathrm{SM}$, the relevant processes are the neutrino-electron scattering and the electron-positron annihilation to photon plus missing energy. The respective $v$-SM cross sections are shown in table 1 . Still not observed coherent neutrino-nuclei scattering, $v+N \rightarrow v+N$, is shown also as a future perspective.

- Step 2: Take an extension to the SM, in this case we will focus in the unparticle scenario. From the phenomenological point of view, the relevant point is that at the low energy regime, there will be an effective interactions for the scalar and vector unparticle operators with the SM fermion fields which are expressed in the effective 
TABLE 2. Cross section mediated by scalar and vectorial unparticle propagators for the $v_{e}+e$ scattering. We have defined $g_{i f}^{\alpha \beta}(d)=\frac{\lambda_{i v}^{\alpha \beta} \lambda_{i f}}{2 \sin (d \pi)} \frac{16 \pi^{5 / 2}}{(2 \pi)^{2 d}} \frac{\Gamma(d+1 / 2)}{\Gamma(d-1) \Gamma(2 d)}, i=0,1$ for scalar and vectorial interaction respectively. Cross section for the single-photon production in electron-positron collisions. Here $A_{d}=\frac{16 \pi^{5 / 2}}{(2 \pi)^{2 d}} \frac{\Gamma(d+1 / 2)}{\Gamma(d-1) \Gamma(2 d)}$.

\begin{tabular}{cc}
\hline Unparticle interaction & Cross section \\
\hline Scalar & $\frac{d \sigma_{\mathscr{O}_{S}}}{d T}=\frac{\left[g_{0 e}^{\alpha \beta}(d)\right]^{2}}{\Lambda^{(4 d-4)}} \frac{2^{(2 d-6)}}{\pi E_{V}^{2}}\left(m_{e} T\right)^{(2 d-3)}\left(T+2 m_{e}\right)$ \\
Vectorial & $\frac{d \sigma_{\mathscr{O} V}}{d T}=\frac{1}{\pi} \frac{\left[g_{1 e}^{\alpha \beta}(d)\right]^{2}}{\Lambda^{(4 d-4)}} 2^{(2 d-5)}\left(m_{e}\right)^{(2 d-3)}(T)^{(2 d-4)}\left[1+\left(1-\frac{T}{E_{v}}\right)^{2}-\frac{m_{e} T}{E_{V}^{2}}\right]$ \\
Interference vectorial & $\frac{d \sigma_{\mathscr{O} V}-S M}{d T}=\frac{\sqrt{2} G_{F}}{\pi} \frac{g_{1 e}(d)}{\Lambda^{(2 d-2)}}\left(2 m_{e} T\right)^{(d-2)} m_{e}\left\{g_{L}+g_{R}\left(1-\frac{T}{E_{V}}\right)^{2}-\frac{\left(g_{L}+g_{R}\right)}{2} \frac{m_{e} T}{E_{V}^{2}}\right\}$ \\
\hline$e^{+}+e^{-} \rightarrow \mathscr{U}+\gamma$ & $\frac{d \sigma_{\gamma \mathcal{H}}}{d x}=\int_{y_{\min }}^{y_{\max }} \frac{A_{d}}{(4 \pi)^{2}}\left(\frac{\lambda_{1 e} e}{\Lambda}\right)^{2}\left[\frac{s(1-x)}{\Lambda^{2}}\right]^{(d-2)} \frac{x^{2}+x^{2} y^{2}+4(1-x)}{x\left(1-y^{2}\right)} d y$ \\
\hline
\end{tabular}

Lagrangian:

$$
\begin{aligned}
& \mathscr{L}_{\mathscr{U}_{S}}\left(\lambda_{0 f}, d, \Lambda\right)=\lambda_{0 f} \frac{1}{\Lambda^{d-1}} \bar{f} f \mathscr{O}_{\mathscr{U}}+\lambda_{0 v}^{\alpha \beta} \frac{1}{\Lambda^{d-1}} \bar{v}_{\alpha} v_{\beta} \mathscr{O}_{\mathscr{U}} \\
& \mathscr{L}_{\mathscr{U}_{V}}\left(\lambda_{1 f}, d, \Lambda\right)=\lambda_{1 f} \frac{1}{\Lambda^{d-1}} \bar{f} \gamma_{\mu} f \mathscr{O}_{\mathscr{U}}^{\mu}+\lambda_{1 v}^{\alpha \beta} \frac{1}{\Lambda^{d-1}} \bar{v}_{\alpha} \gamma_{\mu} v_{\beta} \mathscr{O}_{\mathscr{U}}^{\mu},
\end{aligned}
$$

for the scalar and vectorial interaction. Here $\alpha=e, \mu, \tau$ and $f=e, u, d$. The parameters of the model are therefore $\left(\lambda_{i f}, d, \Lambda\right)$. From now and on, we will fix the energy scale where the theory is invariant to $\Lambda=1 \mathrm{TeV}$.

- Step 3: Compute the physical processes. With the help of the Lagrangian eq. (4), we compute the neutrino-electron scattering mediated by the scalar unparticle and the vector unparticle. For the case of the vector interaction, there is an interference term between the SM model cross section and the unparticle sector. All relevant expressions for $v+e$ scattering are collected in table 2. The $v_{e}+e$ scattering is an example of the role of unparticle as intermediate of a new interaction. But the unparticle stuff can be produced directly at accelerators through for instance $e^{+}+e^{-} \rightarrow \mathscr{U}+\gamma$. In this case, the relevant cross section is computed. (See bottom line of Table 2).

Another interesting experimental proposal is the coherent neutrino nuclei interaction [6]. In this case, if $q R<1$ ( $R$ nuclei radii, $q$ the transfered momentum), the $v$ "sees" the nuclei as a point and scatters coherently on it as a whole. This effect has not been measured yet, but there are some experimental proposal planned to detect this process $[7,8,9]$. The effective Lagrangian 4 would give also contributions to this process and therefore we have considered it too in our analysis. It is shown that if such process is detected it would place strong constraints on unparticle interaction. The corresponding cross sections are collected in Table 3. Note that there is again an interference term between the SM sector and the unparticle sector for the case of a vector intermediate unparticle.

Now it is possible to compute the expected number of events as a function of the free parameters $N_{\text {events }}^{\text {Theo }}\left(\lambda_{i f}, d\right)$ with the help of expressions collected in Tables 2-3. 
TABLE 3. Cross section mediated by scalar and vectorial unparticle propagators for the coherent $v_{e}+N$ scattering.

\begin{tabular}{cc}
\hline Type & Cross section \\
\hline Scalar & $\frac{d \sigma_{\mathscr{O}_{S} N}^{V N}}{d T}=\frac{1}{\Lambda^{(4 d-4)}} \frac{2^{(2 d-6)}}{\pi E_{V}^{2}}\left[g_{0 u}(d)(2 Z+N)+g_{0 d}(d)(Z+2 N)\right]^{2}\left(m_{A} T\right)^{(2 d-3)}\left(T+2 m_{A}\right)$ \\
Vectorial & $\frac{d \sigma_{\mathscr{O}_{V}}^{V N}}{d T}=\frac{2^{(2 d-5)}}{\pi \Lambda^{(4 d-4)}} \frac{m_{A}}{\left(m_{A} T\right)^{(2 d-4)}}\left[g_{1 u}(d)(2 Z+N)+g_{1 d}(d)(Z+2 N)\right]^{2}\left[1+\left(1-\frac{T}{E_{V}}\right)^{2}-\frac{m_{A} T}{E_{V}^{2}}\right]$ \\
SM+U & $\frac{d \sigma_{\mathscr{Q} V}^{V N}-S M}{d T}=\frac{\sqrt{2} 2^{2-1} G_{F}}{\pi} \frac{\left[g_{1 u}(d)(2 Z+N)+g_{1 d}(d)(Z+2 N)\right]}{\Lambda^{(2 d-2)}} \frac{m_{A}\left(g_{V}^{p} Z+g_{V}^{n} N\right)}{\left(m_{A} T\right)^{(d-2)}}\left[1+\left(1-\frac{T}{E_{V}}\right)^{2}-\frac{m_{A} T}{E_{V}^{2}}\right]$ \\
\hline
\end{tabular}
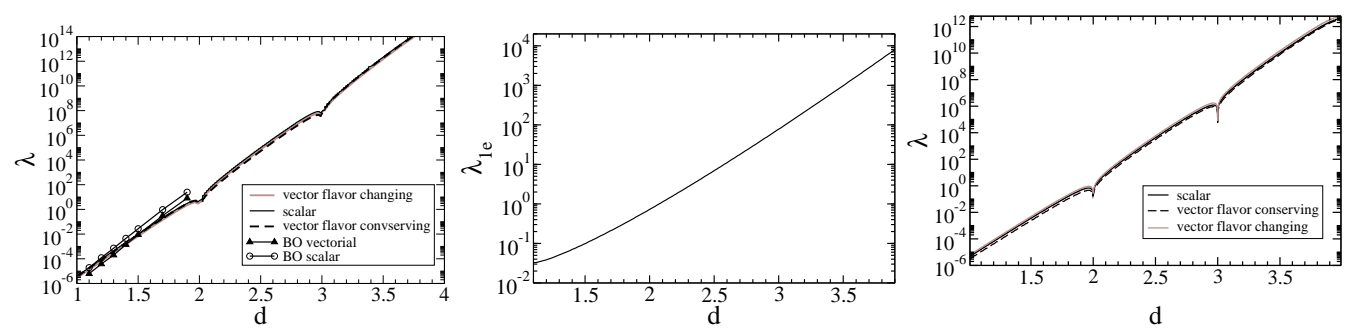

FIGURE 1. Left: Limits on the parameters $d$ and $\lambda_{0,1}=\sqrt{\lambda_{0,1 v}^{e \beta} \lambda_{0,1 e}}(90 \% \mathrm{CL}$ ) from the MUNU experiment for the scalar unparticle case (black solid line) and for the vector unparticle cases, both for flavor changing currents (grey solid line) and for the flavor conserving conserving case (dashed line). Previous bounds obtained by Balantekin and Ozansoy (BO) [11] (dots and triangles) are shown for comparison. The present analysis based on the MUNU data gives stronger constraints on $\lambda_{0,1}$ for values of $d>1.5$. Middle: Limits obtained with LEP data. Right: Sensitivity for unparticle in the coherent $v+N \rightarrow v+N$ scatering TEXONO's proposal.

- Step 4: Now let us compute our $\chi^{2}\left(\lambda_{i f}, d\right)$ where the experimental number of events for the processes collected in table 2 are well known (summarized in [10]). For the case of coherent neutrino-nuclei scattering (Table 3) we will take for definiteness the TEXONO detector [7] neglecting for the moment the resolution function. One more time, for details of the full analysis we refer to [5].

Results: We have obtained the constrains illustrated in Fig. 1 at 90\% C.L. for the scale dimension $d$ and the coupling $\lambda_{0,1}=\sqrt{\lambda_{0,1}^{e \beta} \lambda_{0,1}}$. The values below the lines are allowed. The first two plots show the limits imposed by the current MUNU and LEP data. The third plot shows the sensitivity that an experiment able to detect the coherent neutrino-nuclei scattering.

\section{Astrophysical neutrino and unparticle}

There is another interesting issue related with neutrinos. They are copiously produced in most of the astrophysical objects: the Sun, neutrons star, supernova explosions, merger of Neutron stars, AGN etc, all those astrophysical phenomena involve neutrino production. A very small change in the neutrino properties or interactions will enhance or decrease the number of neutrinos produced due to the large amount of matter and high 
TABLE 4. Constraints on the vector coupling $\lambda_{1}$ from the neutrino electron scattering experiment, and from astrophysical limits.

\begin{tabular}{c|c|c|c|c}
\hline $\mathrm{d}$ & $v-e$ scattering & Eötvös [12] & Long range [13] & SN1987A [14] \\
\hline \hline 1.1 & $2.0 \times 10^{-5}$ & $6.3 \times 10^{-19}$ & $2.8 \times 10^{-23}$ & $9.1 \times 10^{-11}$ \\
1.5 & $9.7 \times 10^{-3}$ & $1.7 \times 10^{-12}$ & $5.7 \times 10^{-12}$ & $5.7 \times 10^{-9}$ \\
2.1 & 40. & $1.1 \times 10^{-2}$ & $6.0 \times 10^{5}$ & $2.9 \times 10^{-6}$ \\
2.5 & $5.5 \times 10^{4}$ & $4.8 \times 10^{4}$ & $1.1 \times 10^{17}$ & $1.8 \times 10^{-4}$ \\
3.1 & $1.2 \times 10^{9}$ & $3.3 \times 10^{14}$ & $1.1 \times 10^{34}$ & $9.9 \times 10^{-2}$ \\
3.5 & $2.1 \times 10^{12}$ & $1.5 \times 10^{21}$ & $3.2 \times 10^{45}$ & 6.1 \\
3.9 & $1.1 \times 10^{15}$ & $6.2 \times 10^{27}$ & $5.8 \times 10^{56}$ & 414.3 \\
\hline
\end{tabular}

energies involved. This fact has been used successfully in order to constrain neutrino properties and/or new interactions. The unparticle case was not the exception and currently there are several limits obtained. In table 4 we show some limits and we include the limits obtained from our analysis. Despite some of the limits are orders of magnitude stronger than the ones obtained by terrestrial experiments, there are assumptions made and, in that respect, a direct measurement always offers a clean determination of free parameters. For a more extensive discussion of the assumptions made see [5].

In summary, we have illustrated how neutrino data can offer a clean way for constraining free parameters of new extensions to the standard model of elementary particles. We used as a test the unparticle scenario and we see that present and future experiments in neutrino physics are competitive and complementary to other high energy and astrophysics observations.

Acknowledges JB thanks the organizing committee of the EAV for the opportunity of giving this talk. This work has been supported by CONACyT, SNI-Mexico and PAPIIT Grant No. IN104208.

\section{REFERENCES}

1. S. M. Bilenky, Phys. Scripta T121, 17 (2005).

2. C. Amsler et al. [Particle Data Group], Phys. Lett. B 667, 1 (2008).

3. H. Georgi, Phys. Rev. Lett. 98, 221601 (2007).

4. H. Georgi, Phys. Lett. B 650, 275 (2007).

5. J. Barranco, A. Bolanos, O. G. Miranda, C. A. Moura and T. I. Rashba, Phys. Rev. D 79, 073011 (2009)

6. J. Barranco, O. G. Miranda and T. I. Rashba, JHEP 0512, 021 (2005).

7. H. T. Wong, H. B. Li, J. Li, Q. Yue and Z. Y. Zhou, J. Phys. Conf. Ser. 39 (2006) 266.

8. K. Scholberg, Phys. Rev. D 73 (2006) 033005. J. Serreau and C. Volpe, Phys. Rev. C 70 (2004) 055502. A. Bueno, M. C. Carmona, J. Lozano and S. Navas, Phys. Rev. D 74 (2006) 033010.

9. P. S. Barbeau, J. I. Collar and O. Tench, JCAP 0709, 009 (2007).

10. J. Barranco, O. G. Miranda, C. A. Moura and J. W. F. Valle, Phys. Rev. D 77, 093014 (2008).

11. A. B. Balantekin and K. O. Ozansoy, Phys. Rev. D 76, 095014 (2007).

12. N. G. Deshpande, S. D. H. Hsu and J. Jiang, Phys. Lett. B 659, 888 (2008).

13. M. C. Gonzalez-Garcia, P. C. de Holanda and R. Zukanovich Funchal, JCAP 0806, 019 (2008).

14. S. Hannestad, G. Raffelt and Y. Y. Y. Wong, Phys. Rev. D 76, 121701(R) (2007). 